\title{
Diagnosing the Treatment
}

The approach to clinical conundrums by an expert clinician is revealed through the presentation of an actual patient's case in an approach typical of a morning report. Similar to patient care, sequential pieces of information are provided to the clinician, who is unfamiliar with the case. The focus is on the thought processes of both the clinical team caring for the patient and the discussant.

This icon represents the patient's case. Each paragraph that follows represents the discussant's thoughts.

Sarah A. McGuffin, MD, MS,2,*, Robert L. Trowbridge, MD,4, Ann M. O'Hare, MD, , and Andrew P. J. Olson, MD7,8,9

'Division of Allergy and Infectious Diseases, University of Washington, Seattle, Washington; ${ }^{2}$ Department of Internal Medicine, University of Washington, Seattle, Washington; ${ }^{3}$ Department of Internal Medicine, Maine Medical Center, Portland, Maine; ${ }^{4}$ Department of Medicine, Tufts University School of Medicine, Boston, Massachusetts; ${ }^{5}$ Division of Nephrology, VA Puget Sound Health Care System, Seattle, Washington; ${ }^{6}$ Division of Nephrology, University of Washington, Seattle, Washington; ${ }^{7}$ Department of Medicine, University of Minnesota, Minneapolis, Minnesota; ${ }^{8}$ Department of Pediatrics, University of Minnesota, Minneapolis, Minnesota; 'University of Minnesota Medical School, Minneapolis, Minnesota.

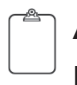

A 70-year-old man presented to the emergency department with 5 days of decreased appetite, frequent urination, tremors, and memory difficulties. He also reported 9 months of malaise, generalized weakness, and weight loss. There was no history of fever, chills, nausea, diarrhea, constipation, pain, or focal neurologic complaints.

This patient exemplifies a common clinical challenge: an older adult with several possibly unrelated concerns. In many patients, a new presentation is usually either a different manifestation of a known condition (eg, a complication of an established malignancy) or the emergence of something they are at risk for based on health behavior or other characteristics (eg, lung cancer in a smoker). The diagnostic process in older adults can be complicated because many have, or are at risk for, multiple chronic conditions.

After reviewing the timeline of symptoms, the presence of nine months of symptoms suggests a chronic and progressive underlying process, perhaps with subsequent superimposition of an acute problem. Although it is not certain whether chronic and acute symptoms are caused by the same process, this assumption is reasonable. The superimposition of acute symptoms on a chronic process may represent progression of the underlying condition or an acute complication of the underlying disease. However, the patient's chronic symptoms of malaise, weakness, and weight loss are nonspecific.

Although malignancy is a consideration given the age of the patient and time course of symptoms, attributing the symp-

\footnotetext{
*Address for correspondence: Sarah A. McGuffin, MD, MS, Division of Allergy and Infectious Diseases, University of Washington, 959 NE Pacific St Health Sciences Building, BB-545 Seattle, WA 98195; Telephone: 206-685-7288; Fax: 206-616-3892; E-mail: mcguffin@uw.edu

Published online first June 27, 2018

Received: October 25, 2017; Revised: March 6, 2018; Accepted: March 13, 2018
}

๑ 2018 Society of Hospital Medicine DOI 10.12788/jhm.2981 toms to a specific pattern of disease or building a cogent differential diagnosis is difficult until additional information is obtained. One strategy is to try to localize the findings to one or more organ systems; for example, given that tremors and memory difficulties localize to the central nervous system, neurodegenerative disorders, such as "Parkinson plus" syndromes, and cerebellar disease are possible. However, this tactic still leaves a relatively broad set of symptoms without an immediate and clear unifying cause.

The patient's medical history included hyperlipidemia, peripheral neuropathy, prostate cancer, and papillary bladder cancer. The patient was admitted to the hospital four months earlier for severe sepsis presumed secondary to a urinary tract infection, although bacterial cultures were sterile. His social history was notable for a $\mathbf{5 0}$ pack-year smoking history. Outpatient medications included alfuzosin, gabapentin, simvastatin, hydrocodone, and cholecalciferol. He used a Bright Light Therapy lamp for one hour per week and occasionally used calcium carbonate for indigestion. The patient's sister had a history of throat cancer.

On examination, the patient was detected with blood pressure of $104 / 56 \mathrm{~mm} \mathrm{Hg}$, pulse of 85 beats per minute, temperature of $98.2{ }^{\circ} \mathrm{F}$, oxygen saturation of $97 \%$ on ambient air, and body mass index of $18 \mathrm{~kg} / \mathrm{m}^{2}$. The patient appeared frail with mildly decreased strength in the upper and lower extremities bilaterally. The remainder of the physical examination was normal. Reflexes were symmetric, no tremors or rigidity was noted, sensation was intact to light touch, and the response to the Romberg maneuver was normal.

Past medical history is the cornerstone of the diagnostic process. The history of two different malignancies is the most striking element in this case. Papillary bladder cancer is usually a local process, but additional information is needed regarding its stage and previous treatment, including whether or not the 
patient received Bacille Calmette Guerin (BCG) vaccine, which can rarely be associated with infectious and inflammatory complications. Metastatic prostate cancer could certainly account for his symptomatology, and bladder outlet obstruction could explain the history of urinary frequency and probable urosepsis. His medication list suggested no obvious causes to explain his presentation, except that cholecalciferol and calcium carbonate, which when taken in excess, can cause hypercalcemia. This finding is of particular importance given that many of the patient's symptoms, including polyuria, malaise, weakness, tremor, memory difficulties, anorexia, acute kidney injury and (indirectly) hypotension and weight loss, are also seen in patients with hypercalcemia. The relatively normal result of the neurologic examination decreases the probability of a primary neurologic disorder and increases the likelihood that his neurologic symptoms are due to a global systemic process. The relative hypotension and weight loss similarly support the possibility that the patient is experiencing a chronic and progressive process.

The differential diagnosis remains broad. An underlying malignancy would explain the chronic progressive course, and superimposed hypercalcemia would explain the acute symptoms of polyuria, tremor, and memory changes. Endocrinopathies including hyperthyroidism or adrenal insufficiency are other possibilities. A chronic progressive infection, such as tuberculosis, is possible, although no epidemiologic factors that increase his risk for this disease are present.

The patient had serum calcium of $14.5 \mathrm{mg} / \mathrm{dL}$, ionized calcium of $3.46 \mathrm{mEq} / \mathrm{L}$, albumin of $3.6 \mathrm{~g} / \mathrm{dL}$, BUN of 62 $\mathrm{mg} / \mathrm{dL}$, and creatinine of $3.9 \mathrm{mg} / \mathrm{dL}$ (all values were normal three months prior). His electrolytes and liver function were otherwise normal. Moreover, he had hemoglobin level of $10.5 \mathrm{mg} / \mathrm{dL}$, white blood cell count of $4.8 \times 10^{\circ} \mathrm{cells} / \mathrm{L}$, and platelet count of $203 \times 10^{9}$ cells/L.

Until this point, only nonspecific findings were identified, leading to a broad differential diagnosis with little specificity. However, laboratory examinations confirm the suspected diagnosis of hypercalcemia, provide an opportunity to explain the patient's symptoms, and offer a "lens" to narrow the differential diagnosis and guide the diagnostic evaluation. Hypercalcemia is most commonly secondary to primary hyperparathyroidism or malignancy. Primary hyperparathyroidism is unlikely in this patient given the relatively acute onset of symptoms. The degree of hypercalcemia is also atypical for primary hyperparathyroidism because it rarely exceeds $13 \mathrm{mg} / \mathrm{dL}$, although the use of concurrent vitamin $D$ and calcium supplementation could explain the high calcium level. Malignancy seems more likely given the degree of hypercalcemia in the setting of weight loss, tobacco use, and history of malignancy. Malignancy may cause hypercalcemia through multiple disparate mechanisms, including development of osteolytic bone metastases, elaboration of parathyroid hormone-related Peptide (PTHrP), increased production of 1,25-dihydroxyvitamin D, or, very rarely, ectopic production of parathyroid hormone (PTH). However, none of these mechanisms are particularly common in bladder or prostate cancer, which are the known malignancies in the patient. Other less likely and less common causes of hypercalcemia are also possible given the clinical clues, including vitamin D toxicity and milk alkali syndrome (vitamin D and calcium carbonate supplementation), multiple endocrine neoplasia (a sister with throat cancer), and granulomatous disease (weight loss). At this point, further laboratory evaluations would be helpful, specifically determination of PTH and PTHrP levels and serum and urine protein electrophoresis.

With respect to the patient's past medical history, his Gleason $3+3$ prostate cancer was diagnosed 12 years prior to admission and treated with external beam radiation therapy and brachytherapy. His bladder cancer was diagnosed 3 years before admission and treated with tumor resection followed by 2 rounds of intravesical BCG (iBCG), 1 round of mitomycin $C$, and 2 additional rounds of iBCG over the course of treatment spanning 2 years and 6 months. The treatment was complicated by urethral strictures requiring dilation, ureteral outlet obstruction requiring left ureteral stent placement, and multiple urinary tract infections.

The patient's last round of iBCG was delivered 6 months prior to his current presentation. The patient's hospital admission 4 months earlier for severe sepsis was presumed secondary to a urologic source considering that significant pyuria was noted on urinalysis and he was treated with meropenem, although bacterial cultures of blood and urine were sterile. From the time of discharge until his current presentation, he experienced progressive weakness and an approximately $50 \mathrm{lb}$ weight loss.

The prior cancers and associated treatments of the patient may be involved in his current presentation. The simplest explanation would be metastatic disease with resultant hypercalcemia, which is atypical of either prostate or bladder cancer. The history of genitourinary surgery could predispose the patient to a chronic infection of the urinary tract with indolent organisms, such as a fungus, especially given the prior sepsis without clear etiology. However, the history would not explain the presence of hypercalcemia. Tuberculosis must thus be considered given the weight loss, hypercalcemia, and "sterile pyuria" of the patient. A more intriguing possibility is whether or not the patient's constellation of signs and symptoms might be a late effect of iBCG. Intravesical BCG for treatment of localized bladder cancer is occasionally associated with complications. BCG is a modified live form of Mycobacterium bovis which invokes an intense inflammatory reaction when instilled into the bladder. These complications include disseminated infection and local complications, such as genitourinary infections. BCG infection might also explain the severe sepsis of unclear etiology that the patient had experienced 4 months earlier. Most interestingly, hypercalcemia has been described in the setting of BCG infection. Diagnosis of disseminated BCG is best made via culture or polymerase chain reaction testing for $M$. bovis at potential sites of involvement, including the blood. Neverthe- 
less, a common presentation of a common disorder is still most likely. If his current presentation is distilled down to a chronic presentation of weakness, weight loss, and hypercalcemia in the setting of known malignancy, then the underlying malignancy seems to offer the most unifying explanation. Given that neither of his known cancers are commonly associated with hypercalcemia, the possibility that he has developed a third malignancy must also be considered.

In the hospital, the patient received intravenous normal saline, furosemide, and pamidronate. Evaluation for hypercalcemia revealed appropriately suppressed PTH ( $8 \mathrm{mg} / \mathrm{dL})$, and normal levels of PTHrP (<.74 pmol/L), prostate specific antigen $(<.01 \mathrm{ng} / \mathrm{mL})$, and morning cortisol $(16.7 \mathrm{mcg} / \mathrm{dL})$. Serum and urine electrophoresis did not show evidence for monoclonal gammopathy, and the 25-hydroxy vitamin $D$ level $(39.5 \mathrm{ng} / \mathrm{mL})$ was within the normal limits (normal range 20.1-50.0 ng/mL). The patient had elevated levels of 1,25-dihydroxy vitamin D (122 ng/ $\mathrm{mL}$, normal range 19.9-79.3 pg/mL), lactate dehydrogenase (196 units/L, normal 50-150 units/L), and angiotensin-converting enzyme (153 units/L, normal 14-82 units/L).

The suppressed PTH level makes primary hyperparathyroidism unlikely, the low PTHrP level decreases the probability of a paraneoplastic process, and the normal protein electrophoresis makes multiple myeloma unlikely. The presence of a significantly elevated 1,25-dihydroxy vitamin D level with a normal 25-hydroxy vitamin $D$ level indicates extrarenal conversion of 25-hydroxy vitamin D by 1-hydroxylase as the etiology of hypercalcemia. Increased activity of 1-hydroxylase is the most consistent with granulomatous diseases, including sarcoidosis, and, with the exception of lymphoma, would not be expected in hypercalcemia malignancy. This mechanism is also associated with tuberculosis, disseminated fungal infections, such as coccidioidomycosis and histoplasmosis, and as a late effect of BCG treatment, regardless of whether disseminated infection or granulomatous immune response. Elevated lactate dehydrogenase and angiotensin-converting enzyme levels may also be noted in many of these disorders.

Lymphoma would appear to be the most likely diagnosis as it accounts for most of the clinical findings observed in the patient and is a fairly common disorder. Sarcoidosis is also reasonably common and would explain the laboratory abnormalities but is not usually associated with weight loss and frailty. Disseminated infections, such as tuberculosis, histoplasmosis, and coccidioidomycosis, are all possible, but the patient lacks key risk factors for these infections. A complication of iBCG is the most intriguing possibility and could account for many of the patient's clinical findings, including the septic episode, which is an event not clearly accounted for by the other diagnostic possibilities. However, disseminated BCG and hypersensitivity reactions to BCG leading to hypercalcemia are rare. When asked to choose between the most interesting possibility and the most common possibility, the most common will usually be the best (and safest) bet. Nonetheless, the effects of prior BCG treatment, including disseminated infection or diffuse immune-mediated granulomatous disease, would be near the top of the differential diagnosis in this case.

The bone survey was normal, the renal ultrasound examination showed nodular wall thickening of the bladder with areas of calcification, and the CT scan of the chest, abdomen, and pelvis showed an area of calcification in the superior portion of the bladder but no evidence of lymphadenopathy or masses to suggest lymphoma. Aerobic and anaerobic blood and urine cultures were sterile. The patient was discharged 12 days after admission with plans for further outpatient diagnostic evaluation. At this time, his serum calcium had stabilized at $10.5 \mathrm{mg} / \mathrm{dL}$ with pamidronate, diuretics, and aggressive oral hydration.

Outpatient bone marrow biopsy revealed a normocellular marrow with multiple small epithelioid granulomas consisting of histiocytes and Touton-type giant cells. Outpatient cystoscopy with barbotage was notable for recurrent urethral stricture that required dilation but did not reveal any new lesions or tumors. At $\mathbf{4 2}$ days after discharge, acid-fast culture and stain from blood cultures obtained in the hospital on day $10 \mathrm{grew}$ acid-fast bacilli of the Mycobacterium tuberculosis complex (Figure). In broth culture, the bacilli were noted to form macroscopic cords. ${ }^{1,2}$ Given the concern for disseminated $M$. bovis, the patient was started on antituberculosis therapy with isoniazid, pyridoxine, rifampin, and ethambutol along with a short course of steroids for presumed granuloma-associated hypercalcemia. The PCR results confirmed that the organism was $M$. bovis. The patient responded well to this course of treatment. His hypercalcemia resolved rapidly, and he regained weight, strength, and energy over the ensuing months.

\section{DISCUSSION}

Hypercalcemia is a common finding in both hospital and ambulatory settings. The classic symptoms associated with hypercalcemia are aptly summarized with the mnemonic "bones, stones, abdominal groans, and psychiatric overtones" (to represent the associated skeletal involvement, renal disease, gastrointestinal symptoms, and effects on the nervous system). However, the severity and type of symptoms vary depending on the degree of hypercalcemia, acuity of onset, and underlying etiology. The vast majority (90\%) of hypercalcemia cases are due to primary hyperparathyroidism and malignancy. ${ }^{3}$ Measuring the PTH level is a key step in the diagnostic evaluation process. An isolated elevation of PTH confirms the presence of primary or possibly tertiary hyperparathyroidism. Low PTH concentrations ( $<20 \mathrm{pg} / \mathrm{mL}$ ) occur in the settings of PTHrP or vitamin-D-mediated hypercalcemia such as hypervitaminosis D, malignancy, or granulomatous disease.

Elevated PTHrP occurs most commonly in squamous cell, renal, bladder, and ovarian carcinomas.,4 Elevated levels of 25-hydroxy vitamin D can occur with excessive consumption of vitamin D-containing products and some herbal supplements. In this case, neither PTHrP nor 25-hydroxy vitamin D level was 

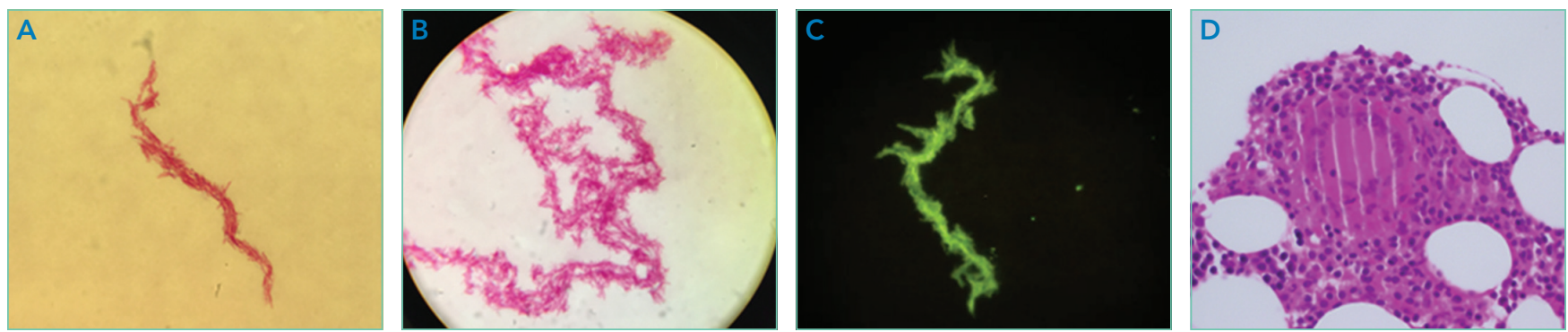

FIG. Blood cultures showing acid-fast bacteria on Kinyoun stain at low (A) and higher (B) power. The mycobacteria bacilli in the patient's cultures assembled in an end-to-end serpentine arrangement known as "cording." Cord formation is characteristic of mycobacteria and due to a specific cell wall glycolipid, namely, trehalose 6, 6'-dimycolate or cord factor, which contributes to mycobacterial virulence, granuloma formation, and humoral and cellular immune responses. The tendency to aggregate in cords actually disappeared in the attenuated form of Mycobacterium bovis cultured by Calmette and Guerin, suggesting that the patient had been exposed to and thus subsequently infected with a more virulent form of BCG. (C) Auramine stain of blood cultures growing Mycobacterium bovis, showing similar serpentine cord formation. (D) Bone marrow biopsy with multiple granulomas consisting of histocytes and Touton-type giant cells consistent with BCG infection.

elevated, leading to an exhaustive search for other causes. Although iBCG treatment is a rare cause of hypercalcemia, 2 previous reports indicated the presence of hypercalcemia secondary to granuloma formation in treated patients. 5,6

The finding of an elevated 1,25-dihydroxy vitamin D level was unexpected. As the discussant mentioned, this finding is associated with lymphoma and with granulomatous disorders that were not initially strong diagnostic considerations in the patient. A variety of granulomatous diseases can cause hypercalcemia. Sarcoidosis and tuberculosis are the most common, but berylliosis, fungal infections, Crohn's disease, silicone exposure, and granulomatosis with polyangiitis may also be associated with hypercalcemia. ${ }^{7}$ The mechanism for hypercalcemia in these situations is increased intestinal calcium absorption mediated by inappropriately increased, PTH-independent, extrarenal calcitriol (1,25-dihydroxy vitamin D) production. Activated monocytes upregulate $25(\mathrm{OH}) \mathrm{D}$-alpha-hydroxylase, converting 25-hydroxy vitamin D to 1,25-dihydroxy vitamin D. Concurrently, the elevated levels of gamma-interferon render macrophages resistant to the normal regulatory feedback mechanisms, thereby promoting the production and inhibiting the degradation of 1,25-dihydroxy vitamin D.8

The tuberculosis vaccine BCG is an attenuated form of $M$. bovis and was originally developed by Albert Calmette and Camille Guérin at the Pasteur Institute in Paris in the early $20^{\text {th }}$ century. In addition to its use as a vaccine against tuberculosis, BCG can protect against other mycobacterial infections, help treat atopic conditions via stimulation of the Th1 cellular immune response, and has been used as an antineoplastic agent. To date, BCG remains the most effective agent available for intravesical treatment of superficial bladder cancer., 10 Although iBCG therapy is considered relatively safe and well-tolerated, rare complications do occur. Localized symptoms (bladder irritation, hematuria) and/or flu-like symptoms are common immediately after instillation and thought to be related to the cellular immune response and inflammatory cascade triggered by mycobacterial antigens. ${ }^{11}$ Other adverse effects, such as infectious and noninfectious complications, may occur months to years after treatment with $B C G$, and the associated symptoms can be quite nonspecific. Infectious complications include mycobacterial prostatitis, orchiepididymitis, balantitis, pneumonia, hepatitis, nephritis, septic arthritis, osteomyelitis, infected orthopedic and vascular prostheses, endocarditis, and bacteremia. Traumatic catheterization is the most common risk factor for infection with BCG. ${ }^{11-13}$ Noninfectious complications include reactive arthritis, hypersensitivity pneumonitis, hemophagocytic lymphohistiocytosis $(\mathrm{HLH})$, and sterile granulomatous infiltration of solid organs.

The protean and nonspecific nature of the adverse effects of iBCG treatment and the fact that complications can present weeks to years after instillation can make diagnosis quite challenging. ${ }^{14}$ Even if clinical suspicion is high, it may be difficult to definitively identify BCG as the underlying etiology because acid fast staining, culture, and even PCR can lead to falsely negative results. ${ }^{14,15}$ For this reason, biopsy and tissue culture are recommended to demonstrate granuloma formation and identify the presence of $M$. bovis.

Although no prospective studies have been conducted to assess the optimal therapy for BCG infection, opinion-based recommendations include cessation of BCG treatment, initiation of at least 3 tuberculostatic agents, and treatment for 3-12 months depending on the severity of the complications. ${ }^{11,14} \mathrm{M}$. bovis is susceptible to isoniazid, rifampin, and ethambutol as well as to fluoroquinolones, clarithromycin, aminoglycosides, and doxycycline; however, this organism is highly resistant to pyrazinamide due to single-point mutation. ${ }^{11,16}$ Interestingly, imipenem is used to treat other nontuberculous mycobacterial diseases, such as those caused by M. abscessus, thereby raising the possibility that the patient's exposure to meropenem during treatment for his prior sepsis may have partially treated an acute infection due to $M$. bovis.

Although treatment with steroids is a standard approach for management of hypercalcemia in other granulomatous disorders and leads to rapid reduction in circulating levels of 1,25-dihydroxy vitamin D and serum calcium., specific evidence has not been established to support its efficacy and effectiveness in treating hypercalcemia and other complications due to M. bovis. ${ }^{17}$ Nevertheless, some experts recommend the use of steroids in conjunction with a multidrug tuberculostatic regimen in cases of septicemia and multiorgan failure due to M. bovis. ${ }^{12,14,18-20}$ 
In summary, this case illustrates the importance of making room in differential diagnosis to include iatrogenic complications. That is, when faced with an unclear diagnosis, the provider should consider common and uncommon immediate and delayed side effects of prior therapies.

\section{TEACHING POINTS:}

- Complications of intravesical BCG treatment include manifestations of granulomatous diseases, such as hypercalcemia.

- When generating a differential diagnosis, medical providers should not only consider the possibility of a new disease process or the progression of a known comorbidity but also the potential of an adverse effect related to prior treatments.

- Medical providers should be wary of accepting previously made diagnoses, particularly when key pieces of objective data are lacking.

Disclosures: The authors have no financial or other conflicts of interest that might bias this work.

\section{References}

1. Geisel RE, Sakamoto K, Russell DG, Rhoades ER. In vivo activity of released cell wall lipids of Mycobacterium bovis bacillus Calmette-Guérin is due principally to trehalose mycolates. J Immunol. 2005;174(8):5007-5015. https:// doi.org/10.4049/jimmunol.174.8.5007.

2. Ryll R, Kumazawa $Y$, Yano I. Immunological properties of trehalose dimycolate (cord factor) and other mycolic acid-containing glycolipids--a review. Microbiol Immunol. 2001;45(12):801-811. https://doi.org/10.1111/j.1348-0421.2001. tb01319.x.

3. Carroll MF, Schade DS. A practical approach to hypercalcemia. Am Fam Physician. 2003;67(9):1959-1966.

4. Goldner W. Cancer-related hypercalcemia. J Oncol Pract. 2016;12(5):426432. https://doi.org/10.1200/JOP.2016.011155.

5. Nayar N, Briscoe K. Systemic Bacillus Calmette-Guerin sepsis manifesting as hypercalcaemia and thrombocytopenia as a complication of intravesical Bacillus Calmette-Guerin therapy. Intern Med J. 2015;45(10):1091-1092. https:// doi.org/10.1111/imj.12876.

6. Schattner A, Gilad A, Cohen J. Systemic granulomatosis and hypercalcaemia following intravesical bacillus Calmette-Guerin immunotherapy. J Intern
Med. 2002;251(3):272-277. https://doi.org/10.1046/j.1365-2796.2002.00957.x

7. Tebben PJ, Singh RJ, Kumar R. Vitamin D-mediated hypercalcemia: mechanisms, diagnosis, and treatment. Endocr Rev. 2016;37(5):521-547. https://doi org/10.1210/er.2016-1070.

8. Nielsen $\mathrm{CT}$, Andersen $\AA \mathrm{B}$. Hypercalcemia and renal failure in a case of disseminated Mycobacterium marinum infection. Eur J Intern Med. 2016;20(2):e29-e31. https://doi.org/10.1016/j.ejim.2008.08.015.

9. Sylvester RJ. Bacillus Calmette-Guérin treatment of non-muscle invasive bladder cancer. Int J Urol. 2011;18(2):113-120. https://doi.org/10.1111/j.14422042.2010.02678.x.

10. Clark PE, Spiess P, Agarwal N, Al. E. NCCN Guidelines $®$ Insights Bladder Cancer, Version 2.2016 Featured Updates to the NCCN Guidelines. J Natl Compr Canc Netw. 2016;14(10):1213-1224. https://doi.org/10.6004/ jnccn.2016.0131.

11. Decaestecker K, Oosterlinck W. Managing the adverse events of intravesical bacillus Calmette-Guérin therapy. Res Reports Urol. 2015;7:157-163. https:// doi.org/10.2147/RRU.S63448.

12. Gandhi NM, Morales A, Lamm DL. Bacillus Calmette-Guerin immunotherapy for genitourinary cancer. BJU Int. 2013;112(3):288-297. https://doi. org/10.1111/j.1464-410X.2012.11754.X.

13. Brausi M, Oddens J, Sylvester R, et al. Side effects of bacillus calmette-guerin $(B C G)$ in the treatment of intermediate- and high-risk Ta, T1 papillary carcinoma of the bladder: Results of the EORTC genito-urinary cancers group randomised phase 3 study comparing one-third dose with full dose and 1 year with 3 years of maintenance BCG. Eur Urol. 2014;65(1):69-76. https://doi. org/10.1016/j.eururo.2013.07.021.

14. Gonzalez OY, Musher DM, Brar I, et al. Spectrum of bacille Calmette-Guérin (BCG) infection after intravesical BCG immunotherapy. Clin Infect Dis. 2003;36(2):140-148. https://doi.org/10.1086/344908.

15. Pérez-Jacoiste Asín MA, Fernández-Ruiz M, López-Medrano $F$, et al. Bacillus Calmette-Guérin (BCG) infection following intravesical BCG administration as adjunctive therapy for bladder cancer. Medicine (Baltimore) 2014;93(17):236-254. https://doi.org/10.1097/MD.0000000000000119.

16. Durek C, Rüsch-Gerdes S, Jocham D, Böhle A. Sensitivity of BCG to modern antibiotics. Eur Urol. 2000;37(Suppl 1):21-25. https://doi. org/10.1159/000052378.

17. Sharma OP. Hypercalcemia in granulomatous disorders: a clinical review. Curr Opin Pulm Med. 2000;6(5):442-447. https://doi.org/10.1097/00063198200009000-00010.

18. LeMense GP, Strange C. Granulomatous pneumonitis following intravesical BCG: what therapy is needed? Chest. 1994;106(5):1624-1626. https://doi org/10.1378/chest.106.5.1624

19. Nadasy KA, Patel RS, Emmett M, et al. Four cases of disseminated Mycobacterium bovis infection following intravesical BCG instillation for treatment of bladder carcinoma. South Med J. 2008;101(1):91-95. https://doi.org/10.1097/ SMJ.0b013e31815d4047.

20. Macleod LC, Ngo TC, Gonzalgo ML. Complications of intravesical bacillus calmette-guérin. Can Urol Assoc J. 2014;8(7-8):E540-E544. https://doi org/10.5489/cuaj.1411. 